\title{
Long-Term Follow-Up after Paediatric Kidney Transplantation and Influence Factors on Graft Survival: A Single-Centre Experience of 16 years
}

\author{
Frank Friedersdorff $^{\mathrm{a}}$ Therese-Marie Koch ${ }^{\mathrm{b}}$ Beatriz Banuelos-Marco ${ }^{\mathrm{a}}$ \\ Ricardo Gonzalez $^{\mathrm{a}}$ Tom Florian Fuller ${ }^{\mathrm{a}}$ Stefanie von Mechow ${ }^{\mathrm{a}} \quad$ Dominik Müller $^{\mathrm{b}}$ \\ Anja Lingnau ${ }^{a}$
}

a Department of Urology and Pediatric Urology, Charité - Universitätsmedizin Berlin, Corporate Member of Freie Universität Berlin, Humboldt-Universität zu Berlin, and Berlin Institute of Health, Berlin, Germany; ${ }^{\mathrm{b}}$ Department of Pediatric Nephrology, Charité - Universitätsmedizin Berlin, Corporate Member of Freie Universität Berlin, HumboldtUniversität zu Berlin, and Berlin Institute of Health, Berlin, Germany

\section{Keywords}

Influence factors · Paediatric transplantation · Vascular complications

\footnotetext{
Abstract

Introduction: To evaluate graft- and patient survival after paediatric kidney transplantation and detecting influence factors, which affect the post-transplant time. Materials and Methods: We analysed long-term survival rates and complications after paediatric kidney transplantation and searched for predictive parameters for graft function. Results: In 132 patients, 143 kidney transplantations were performed. Graft failure occurred in $25 \%$. Chronic rejections were the leading cause of graft loss (42.9\%). Graft survival rates were $92.2 \%$ after 1 year, $85.5 \%$ after 5 years, $71.1 \%$ after 10 years and $62.1 \%$ after 15 years. The following parameters strongly influenced graft survival: number of transplants ( $p=0.014)$,
}

year of transplant ( $p<0.0001$ for 1997-2005), Epo-therapy post-transplant $(p=0.001)$, hypotension donor $(p=0.027)$, cold ischemia time $(p=0.023)$, anastomosis time $>50 \mathrm{~min}$ $(p=0.008)$, delayed graft function $(p=0.003)$ and deceased donation ( $p=0.039$ ). The percentage of patients who died was $5.6 \%$. Overall patient survival rates were $99.3 \%$ after 1 year, $95.2 \%$ after 5 years, 94.2\% after 10 years and $90.7 \%$ after 15 years. Various types of infections (42.9\%) were the main causes of death. Conclusions: The main causes of death after kidney transplantations in paediatric recipients are malignancy and infections. To avoid vascular complications especially in young recipients (<9 years), the cold ischemia time should be as short as possible.

(c) 2018 S. Karger AG, Basel

\section{KARGER}

(c) 2018 S. Karger AG, Basel 


\section{Introduction}

Renal transplantation remains the treatment of choice for children with end-stage renal disease (ESRD). It confers improved survival, growth and health-related quality of life compared to dialysis [1-3]. Due to new immunosuppressive medication, a decreased number of rejections and improvement of transplant- and patient outcome is seen. Since children receiving kidney transplants have a long expectancy of life, it is particularly important to maximize graft function and graft survival in this population. Many times, children's growth and development are already impaired in early stages of chronic renal disease. Forty percent of children with end-stage renal failure are situated below the 3 rd percentile in growth curves [4]. Kidney transplantation can be considered the best therapy for ESRD. There are about 3,000 renal transplantations performed per year [5]. The mortality rate is $4-5$ fold higher when dialysis is performed compared to organ transplantation [1].

Chronic transplant rejection is the most common reason for graft loss among children [4]. Further reasons for chronic graft dysfunction are acute rejection, toxicity following the application of calcineurin inhibitors, recurrence of the underlying renal disease process within the graft as well as cases of de-novo glomerulonephritis [4].

In literature, the 5 -year graft survival is $85 \%$ for living donation recipients and $78 \%$ for deceased donation recipients [6]. The overall patient survival has been constantly improved within the past years. Smith et al. [7] reported 3 -year survival rates of $95 \%$ for living donation recipients and $93 \%$ for deceased donation recipients. Infections, neoplasia and cardiopulmonary diseases continue to be the most common reasons for recipients' death [6].

Periods awaiting the transplantation, followed by extended duration of renal replacement therapy, are known to negatively influence the graft function and patient survival. Pre-emptively performed transplantations therefore lead to improved graft survival [8]. Approximately $20 \%$ of transplantations in children are performed preemptively [8].

Arterial hypertension significantly increases the risk for cardio-vascular morbidity and mortality. The prevalence of hypertension following kidney transplantation is about $50-80 \%$, the aetiology being multifactorial [9]. The most common reasons for hypertension are presence of diseased native kidneys, pre-existing hypertension, renal artery stenosis as well as chronic allograft nephropathy [10]. Left ventricular hypertrophy is a com- mon result of end-organ damage in transplanted children [10]. Persisting growth retardation is associated with a worse medical outcome such as higher risk of re-hospitalization and has a major impact on the patients' quality of life [11]. To achieve improved results concerning the patients' growth, pre-emptive transplantation should be considered in addition to growth hormone treatment and application of new immunosuppressive drugs followed by long-term immunosuppression without steroid intake [2]. Post-transplant growth spurts can be observed as physiological reaction in children of all ages within the first 6 months following transplantation [6]. Basic reason is steroid intake as well as the ending of alimentary restrictions. Obesity, however, increases the risk in cardiovascular disease and is considered an independent major risk factor for decrease in graft function [12]. The objective is therefore to initiate steroid-sparing protocols and as well as regular blood pressure assessments. Similarly, the nutritional intake should be optimized and patients should be encouraged to physical activities. With a prevalence of $33 \%$ post transplantation, urinary infections are considered an additional factor influencing the outcome of transplant recipients [13]. Basic risk factors could be identified such as urinary tract malformations, use of immunosuppressive drugs and urinary catheters [13]. Regular control and treatment for urinary infection are crucial to minimize the risk of decrease in long-term graft function [13].

The aim of the present study was to evaluate the patient- and transplant outcomes over the time and to identify factors associated with poor outcomes.

\section{Materials and Methods}

The entire analysis was performed with regard to the terms of the Charité Medical University of Berlin ensuring correct scientific research work ("Good Scientific Practice," version 06/10/14). The present study is a retrospective analysis and all patient data are anonymized.

Our database included paediatric renal transplant recipients up to 21 years of age from January 1997 to December 2013. Recipients, who were transplanted at other centres, but treated in our hospital, were excluded from this study. The following preoperative parameters were evaluated: patients and donors demographic data (age, body weight and height, donors condition (e.g., hypotonic episodes), patients primary disease, type and duration of dialysis, previous operations, type of transplant, number of transplant, infectious parameters such as EBV status and pre-transplant transfusions.

We evaluated intraoperative parameters such as operating technique, intraoperative complications, ischemia time and postoperative parameters as function of transplant, immunosuppres- 
sive therapy, rejection episodes, and further developmental parameters of the transplanted patient, such as re-hospitalization, medication, blood pressure, serum creatinine, compliance. For evaluating the data, we used information from hospital archive, Eurotransplant and SAP system. Furthermore, we used an onlinecalculator (www.4c-study.org-calculators) for calculating height age, height SD score, body mass index, body mass index SD score for height age.

\section{Statistical Analyses}

Statistical analyses were performed with SPSS 22 (IBM Corporation, Somers, NY, USA). Several non-parametric tests (MannWhitney U test, Kruskal-Wallis test) were performed. Categorical variables were evaluated using the chi-square test. Predictors of graft survival were analysed by multivariable adjusted logistic regression. A $p$ value of less than 0.05 was considered significant.

\section{Results}

\section{Patient Characteristics}

In the present study, 143 paediatric transplants were included $-47 \%$ girls and $53 \%$ boys. The mean age at transplant was 11.5 years, with $18 \%$ of the children being younger than 5 years at the age of transplant. The most common diagnosis was "congenital anomalies of the kidney and urinary tract" and other complex malformations associated with renal failure $(34.1 \%)$ followed by glomerulopathies (22.7\%), haemolytic uremic syndrome (13.5\%), tubulopathies (12.9\%) and renal failure of other reasons. Living donation was performed in $24.5 \%$ of the cases.

\section{Graft and Patient Survival}

Overall mortality was 5.6\%. Overall survival rate was 99.3, 95.2, 94.2, and 90.7\% after 1, 5, 10, and 15 years respectively. Causes of death were post-transplant lymphoproliferative disease (PTLD) $28 \%$, myocarditis 28\%, sepsis $14 \%$, suicide $14 \%$ and traumatic events $4 \%$.

Graft survival rates were 92.2, 85.5, 71.1, and $62.1 \%$ after $1,5,10$, and 15 years respectively.

Causes of graft failure were chronic rejection $43 \%$, acute rejection $11.4 \%$, vascular problems $14.3 \%$, infections $5.7 \%$, recurrence of primary disease $8.6 \%$ and graft failure of unknown origin $7.1 \%$.

Within the period 1997-2005, graft failure was significantly higher compared with the period 2006-2013 (35.8 vs. $2.1 \%, p<0.001)$. Graft failure within the first year after transplant occurred frequently in the period 1997-2005, but it was not significant $(p=0.054)$. The graft survival was significantly different in favour of period 2006-2013 (5-year graft survival 95 vs. 80\%, $p<0.001$ ).

Long-Term Follow-Up after Paediatric

Kidney Transplantation

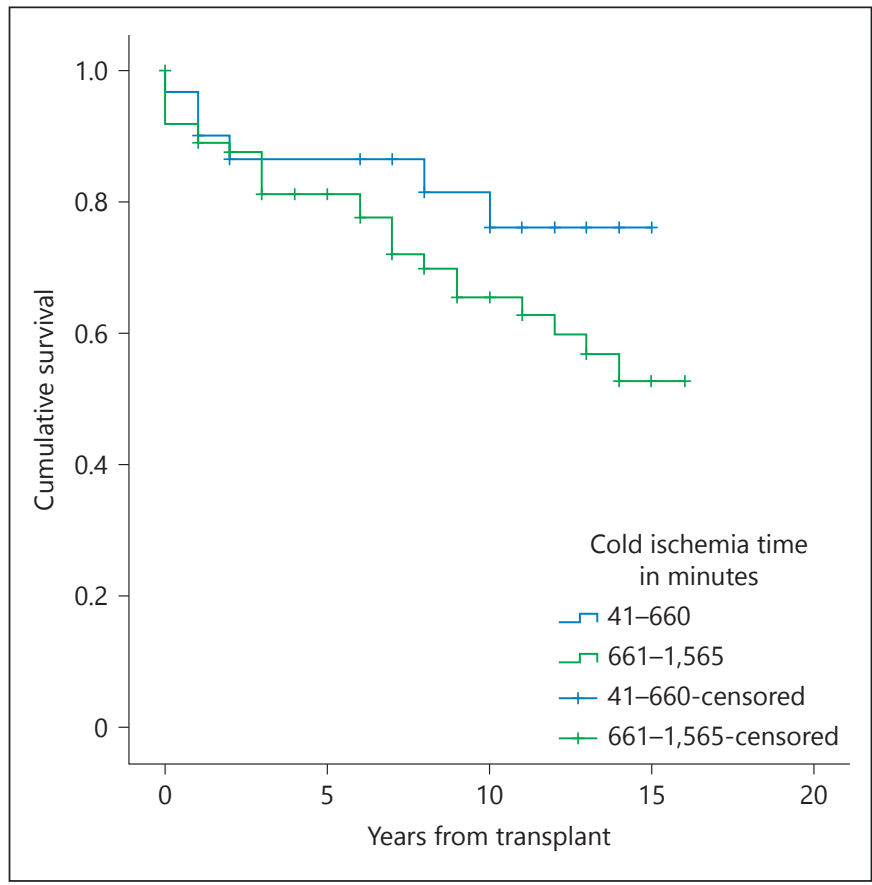

Fig. 1. Cumulative graft survival depends on cold ischaemia time.

\section{Influence Factors on Graft Survival}

Several significant factors that depended on graft survival could be identified. The following factors are significant on graft survival: number of transplants $(p=0.014)$, year of transplant $(p<0.0001$ for 1997-2005), Epo-therapy post-transplant $(p=0.001)$, hypotension donor $(p=$ $0.027)$, cold ischaemia time ( $p=0.023)$, anastomosis time $>50 \min (p=0.008)$, delayed graft function $(p=0.003)$ and deceased donation $(p=0.039)$. Figure 1 shows that the cumulative graft survival depends on cold ischaemia time. The comparison of living and deceased donation regarding graft and patient survival showed a statistically significant difference $(p=0.039)$. Figure 2 shows a KaplanMeier-Curve regarding cumulative graft survival.

The most common reason for graft failure was chronic graft failure or graft dysfunction (42.9\%). Other reasons were vascular complications such as arterial and vein thrombosis or arterial stenosis (14.3\%) or acute rejections (11.4\%). Infectious reasons for graft loss were BK-virus and myocarditis. The mean age at the time of graft failure was $16.1 \pm 6.7$.

\section{Surgical Complications}

Intra- and postoperative complications occurred in $55.2 \%$ of the cases. Within the complication group, a graft failure was $30.4 \%$ and more often seen compared 


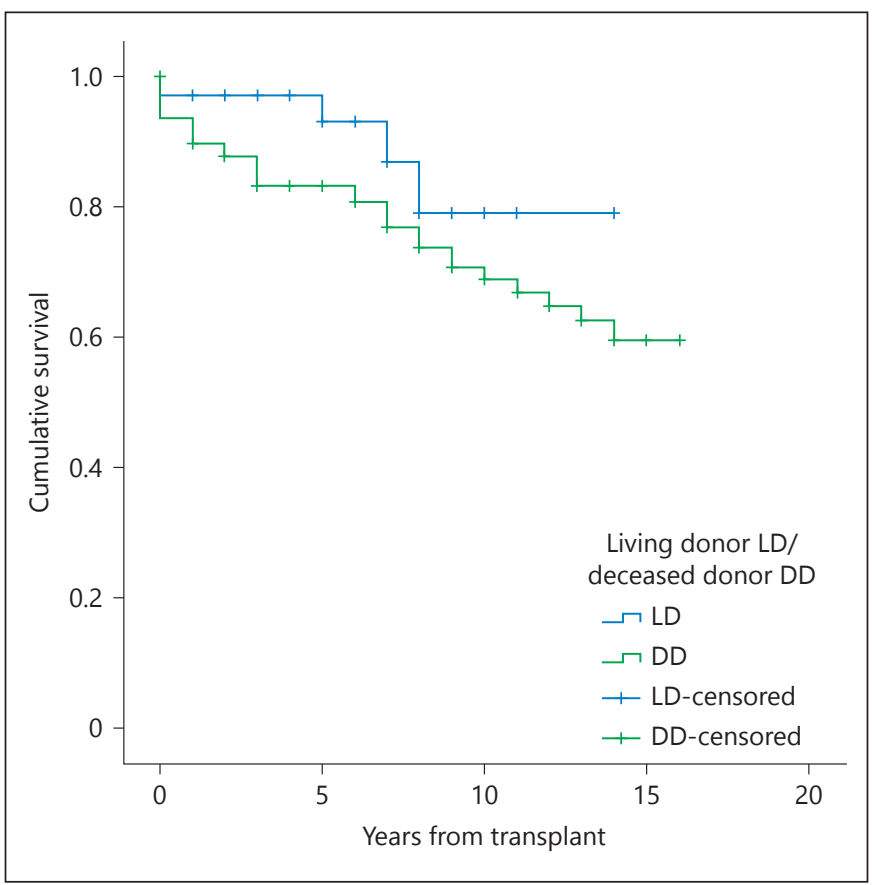

Fig. 2. Cumulative graft survival depends on living or deceased donation.

to the non-complication group ( $p=0.068)$. The most common complications were vascular events (29.3\%). Renal artery stenosis occurred in $9.1 \%$ of all recipients. Renal artery thrombosis and renal vein thrombosis occurred each at the rate of $1.4 \%$. All recipients developing arterial or venous thrombosis experienced graft failure. Further complications were urological type (20.3\%), lymphocele (19.5\%), bleeding (12\%), infections (7.5\%) and abdominal complications (12.5\%) such as ileus and subileus.

\section{Discussion}

Kidney transplantation plays an important role for children with ESRD. Through the transplant, the children have the chance to complete a healthy development of the kidneys. Furthermore, they have a significant survival advantage in comparison to dialysis $[1,14]$. Both for the living donation as well as for deceased donation, within the last decades, the graft survival could be improved $[6,15]$. Smith et al. [6] showed an improved graft survival for the era 2003-2010 in comparison to 1987-2002. The present study showed equally results for deceased donation, partly better graft survival rates for living donation in comparison to Smith et al. [6]. The share of living donations in comparison to other studies was the same or slightly higher $[5,16]$. The overall short- and long-term graft survival after living donation was comparable to that reported in the literature [16].

Vascular thromboses are one of the most common causes of transplant losses after chronic transplant rejection [7]. The present study confirmed this statement. Thromboses occurred with a frequency of $2.8 \%$. All patients experienced a graft failure. There are various risk factors for the occurrence of transplant thromboses. The following factors can have an influence: deceased donation, cold ischaemia time $>24 \mathrm{~h}$, multiple transplants, peritoneal dialysis, more than 5 blood transfusions, donor age or recipients $<6$ years, thrombophilia and low blood pressure during transplantation $[4,7$, 17]. In order to avoid thromboses or renal artery stenosis, especially in cases of young recipients (age $<9$ years), the cold ischaemia time should be as short as possible. In our study, these complications occurred in cases of young recipients (age $<9$ years) and with a cold ischemia time $>24 \mathrm{~h}$. Mehrabi et al. [16] showed that severe hypotension of the donor is a risk factor for hypoxic damage of the renal graft. The present study could confirm this hypothesis. "Hypotension donor" was a significant influencing factor on graft survival. It seems to affect the long-term graft survival and favoured a chronic transplant failure. The 1- and 5-year graft survival (95 and 89\%) was slightly better compared with the literature [16]. The difference could be attributed to the fact that the comparative study dates back to 2004 or a more recent period (1967-2003) [16]. If you look at only the living donor recipients in more recent literature, the graft survival rates are better (98.4\% 1-year, $94.2 \% 5$-year) [6]. The graft survival of deceased donation was comparable with the literature [6]. The deathcensored graft survival after 15 years of follow-up was almost $60 \%$. Studies showed that the 3-year graft survival of children by deceased donation has improved from $78.5 \%$ in the period from $1987-1995$ to $92.8 \%$ in 1996 and from 89.8 to $94.8 \%$ in living donation [7]. Within the investigated patient group, the 3-year patient survival was 96.8 vs. $97.9 \%$ for the 1997-2005 period vs. 2005-2013. It was also comparable or slightly higher compared with literature [7]. The most common causes of death are infections, cardiopulmonary causes and malignant diseases, with nearly half of the patients dying with a functioning graft [6]. Infections, followed by the PTLD, were the most frequent causes of death according to the present study, so that this finding could be confirmed. Contrary to the above-mentioned 
study, the proportion of children who died with a functioning transplant was less than $50 \%$. The statement in the literature that the PTLD represents a significant mortality factor among kidney transplanted children could be confirmed $[7,18]$. The incidence of PTLD was comparable to that reported in other studies of 6.9 and $4.4 \%[19,20]$. The median time after kidney transplantation up to the onset of illness differs strongly in the literature with information between 7.2 months and 3.2 years $[19,20]$. In our study, we found a value of 1.7 years. The increased incidence of PTLD among children who received growth hormone therapy prior to kidney transplantation is controversially discussed in the literature [21]. Within the investigated patient group, no correlation could be detected, whereby the overall small number of cases of children affected by a PTLD must be considered. As an alternative to dialysis, AB0-incompatible kidney transplantation should also be considered as an effective treatment with acceptable incidence of developing malignant tumours or infections [22].

\section{Conclusion}

The main causes of death after kidney transplantations in paediatric recipients are malignancy and infections. Long-term problems are hypertension, cardiovascular diseases, development of growth and weight, urinary tract infections and malignancy. To avoid vascular complications especially in young recipients ( $<9$ years), the cold ischaemia time should be as short as possible.

\section{Disclosure Statement}

All authors declare that they have no competing interests.

\section{References}

1 McDonald SP, Craig JC: Long-term survival of children with end-stage renal disease. N Engl J Med 2004; 350: 26542662.

- 2 Nissel R, Brázda I, Feneberg R, et al: Effect of renal transplantation in childhood on longitudinal growth and adult height. Kidney Int 2004;66:792-800.

3 Goldstein SL, Graham N, Burwinkle T, et al: Health-related quality of life in pediatric patients with ESRD. Pediatr Nephrol 2006;21: 846-850.

4 Mehls O, Scharer K: Padiatrische Nephrologie. Berlin, Springer, 2002.

5 Offner G: [Pediatric kidney transplantation]. Urologe A 2009;48:1464-1467.

-6 Smith JM, Martz K, Blydt-Hansen TD: Pediatric kidney transplant practice patterns and outcome benchmarks, 1987-2010: a report of the North American Pediatric Renal Trials and Collaborative Studies. Pediatric Transplant 2013;17:149-157.

7 Smith JM, Fine RN, McDonald RA: Current state of pediatric renal transplantation. Front Biosci 2008;13:197-203.

${ }_{8}$ Tönshoff B, David-Neto E, Ettenger R, et al: Pediatric aspects of therapeutic drug monitoring of mycophenolic acid in renal transplantation. Transplant Rev (Orlando) 2011; 25:78-89.
-9 Mitsnefes MM: Hypertension and end-organ damage in pediatric renal transplantation. Pediatric Transplant 2004;8:394-399.

10 Seeman T:Hypertension after renal transplantation. Pediatric Nephrol 2007;24:959-972.

-11 Harambat J, Cochat P: Growth after renal transplantation. Pediatric Nephrol 2008;24: 1297-1306.

12 Vester U, Schaefer A, Kranz B, et al: Development of growth and body mass index after pediatric renal transplantation. Pediatric Transplant 2005;9:445-449.

13 John U, Kemper MJ: Urinary tract infections in children after renal transplantation. Pediatric Nephrol 2008;24:1129-1136.

14 Gillen DL, Stehman-Breen CO, Smith JM, et al: Survival advantage of pediatric recipients of a first kidney transplant among children awaiting kidney transplantation. Am J Transplant 2008;8:2600-2606.

15 Gulati A, Sarwal MM: Pediatric renal transplantation: an overview and update. Curr Opin Pediatr 2010;22:189-196.

16 Mehrabi A, Kashfi A, Tönshoff B, et al: Longterm results of paediatric kidney transplantation at the University of Heidelberg: a 35 year single-centre experience. Nephrol Dial Transplant 2004;19(suppl 4):iv69-iv74.

17 Oh J, Schaefer F, Veldmann A, et al: Heterozygous prothrombin gene mutation: a new risk factor for early renal allograft thrombosis. Transplantation 1999;68:575-578.

18 Dharnidharka VR, Sullivan EK, Stablein DM, et al: North American Pediatric Renal Transplant Cooperative Study (NAPRTCS). Risk factors for post-transplant lymphoproliferative disorder (PTLD) in pediatric kidney transplantation: a report of the North American Pediatric Renal Transplant Cooperative Study (NAPRTCS). Transplantation 2001;71:10651068.

19 McDonald RA, Smith JM, Ho M, et al: Incidence of PTLD in pediatric renal transplant recipients receiving basiliximab, calcineurin inhibi-tor, sirolimus and steroids. Am J Transplant 2008;8:984-989.

20 Cleper R, Ben Shalom E, Landau D, et al: Posttransplantation lymphoproliferative disorder in pediatrickidney-transplant recipients - a national study. Pediatr Transplant 2012; 16:619-626.

21 Dharnidharka VR, Talley LI, Martz KL, et al: Recombinant growth hormone use pretransplant and risk for post-transplant lymphoproliferative disease - a report of the NAPRTCS. Pediatr Transplant 2008;12: 689-695.

22 Uchida J, Iwai T, Kabei K, et al: AB0-incompatible living kidney transplant recipients from spousal donors receiving rituximab. Urol Int 2016;97:457-465. 